\title{
Defining heterogeneity as a second level of variation
}

\author{
B. B. Hanberry \\ Department of Forestry, University of Missouri, 203 Natural Resources Building, Columbia, MO 65211, USA \\ Correspondence to: B. B. Hanberry (hanberryb@missouri.edu)
}

Received: 15 January 2015 - Revised: 11 May 2015 - Accepted: 01 June 2015 - Published: 19 June 2015

\begin{abstract}
The term heterogeneity has been defined in various ways so that the meaning of heterogeneity has become ambiguous. However, heterogeneity can be defined carefully as a distinct response to multiple single types of underlying variation, that is, a secondary level of variation (or "metavariation"). Identification of heterogeneity is affected by multiple factors, including researcher decisions, and ecosystems at a specified scale can contain both heterogeneous and homogenous variables. A formalized definition may also reduce the suggestion that heterogeneity is more beneficial than homogeneity.
\end{abstract}

\section{Introduction}

Ecological definitions often can not be both generalized and fit specific studies that vary in time, location, extent, taxa, history, disturbance, land use, techniques, measurements, objectives, perspectives, and other factors that present context. In addition, ecological terms with inconsistent use (Hall et al., 1997; Lindenmayer and Fisher, 2007; Madin et al., 2008; McInerny and Etienne, 2012) hinder communication about the concept. Heterogeneity is one such term that may make sense as applied in each particular case but not consistently as a whole. Heterogeneity often has a unique definition in each study, even if the authors have not explicitly stated their definition (Li and Reynolds, 1995). Kolasa and Rollo (1991) recognized wide use of the term heterogeneity and definitional elusiveness despite apparent simplicity. Rather than an exhaustive literature survey, my objective is to initiate a discussion on a well-used ecological term, heterogeneity, that may be of interest to a broad audience, by proposing a more limited definition that is free of value judgments.

\section{Why ambiguous terminology is a problem}

Variation is a hallmark of ecology, which eventually introduces ambiguity into concepts and terminology. Ambiguity creates misdirection and confusion in interpretation, reduces comparability and detection in literature searches for related research, and prevents effective and efficient use and understanding of terms (Madin et al., 2008; McInerny and Eti- enne, 2012). Use of heterogeneity to invoke different themes may have resulted in a panchreston, in that researchers can not readily understand what heterogeneity means to other researchers and the implications of their research (Lindenmayer and Fisher, 2007). It is symptomatic that Stein et al. (2014) needed a figure to explain heterogeneity because there are hundreds of terms, which often are not defined, unique to each researcher, or used synonymously instead of in a controlled manner. When there is no consensus about when a term should be used, there should be some critical response suggesting that researchers carefully select and define their terminology along with proposals for formalizing use of terminology to improve clarity and communication.

There are numerous publications that have produced varying definitions of heterogeneity, more than one book can cover comprehensively (e.g., Kolasa and Pickett, 1991; Hutchings et al., 2000). Heterogeneity may have originated in reaction to the idea that nature was stable, orderly, constant, and homogeneous (McIntosh, 1991; Weins, 2000). Heterogeneity initially was differentiated from variation by including more than one variable (i.e., heterogeneity defined as composition of parts of different kinds; Kolasa and Rollo, 1991). However, heterogeneity often appears to be used interchangeably with variation (i.e., measurement of range of one variable; Fahrig et al., 2011), diversity (i.e., species' richness and distribution, particularly using diversity metrics; Malanson and Cramer, 1999), dynamics (i.e., temporal dynamics), gradients (e.g., elevational, topographical, soil, climate, resource, nutrient; Stein et al., 2014), complexity, and 
landscape metrics (e.g., continuous, patchy, configuration, composition; Stein et al., 2014). Rees et al. (2000) defined heterogeneity as (1) variation around a parameter, (2) abiotic and biotic variables that cause variability (in population demography), and (3) spatial and temporal variation in the abiotic and biotic environment. Furthermore, there are multiple types of heterogeneity: spatial, temporal, functional, measured, hierarchical, habitat, environmental, population, and organizational (Kolasa and Rollo, 1991); landscape, compositional, configural, and structural (Fahrig et al., 2011); genetic and taxonomic (Olden and Rooney, 2006); non-specific and individual-specific (Rees et al., 2000); surface reflectance, spatio-spectral, wavelength, and nested (for land cover and other remotely sensed variables; Lausch et al., 2013); biogenic and physical (Pickett et al., 2000); statistical and network. Heterogeneity is also used outside of ecology, in fields ranging from health to economics.

\section{Limiting the definition of heterogeneity}

Consistent and formalized terminology will reduce the "heterogeneity of heterogeneity" (Kolasa and Rollo, 1991) and perhaps require that at minimum, researchers are more thoughtful in the use of the term heterogeneity. The term variation should be used for all unspecified variation. Heterogeneity arises from comparisons of different sources of variation that occur in space, time, as interactive networks or contacts (e.g., plant-pollinator; Marsh et al., 2011; Rosenberg and Freedman, 1994), or as studies for meta-analysis. Because variation occurs independently within a sample, while heterogeneity results from comparing samples, heterogeneity is additive or emergent as a secondary level to variation. Instead of being multivariate (including more than one variable; Kolasa and Rollo, 1991), heterogeneity is "metavariate", arising from underlying multiple sources of variation. For example, heterogeneity appears to be most typically explicit for change over space; thus, heterogeneity emerges in comparison among spatial units instead of within spatial units (Fig. 1). Usually there is a reasonable boundary in space or time to separate samples that match justifiably with the variable of interest.

Generally, abiotic variables present as the primary level of variation and as such, use of the term "variation" instead of "heterogeneity" will be more appropriate. In particular, although abiotic conditions (McInerny and Etienne, 2012) of topography or elevation may be sources of heterogeneity, these variables are static or slow to change over time (in a human timeframe) and do not respond to other sources of variation. However, water and soil are responsive abiotic variables; these resources are affected by variation in plants and insects (Tsunoda et al., 2014) and thus, heterogeneity can be an appropriate term.

Homogeneity represents lack of response when comparing primary units of variance. Homogeneity has also become

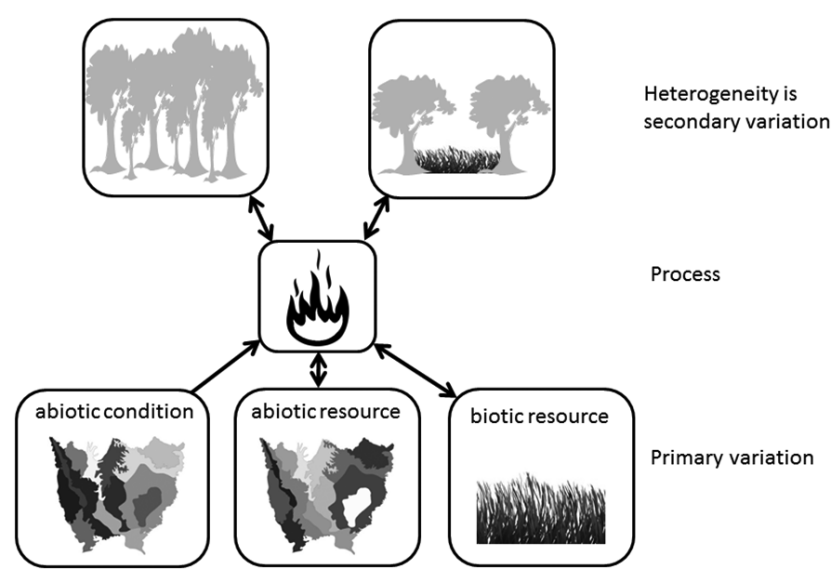

Figure 1. In this example, primary variation occurs in abiotic and biotic variables. Abiotic conditions, such as topography, will affect other variables but generally will not respond to other variables, while abiotic resources, such as water, and biotic resources, such as herbaceous vegetation that provides fine fuels, will interact with other variables. A process, such as fire, may be necessary for some interactions to create a secondary level of response, for example by vegetation in the form of varying phases of forest ecosystems. A heterogeneous continuum of savannas to woodlands to closed forests develops due to different exposure to fire, based on underlying primary levels of topography, moisture, and fine fuels.

accepted terminology for decreased genetic, taxonomic, or functional diversity (Olden and Rooney, 2006). Diversity is richness and evenness of species; because evenness has a spatial component and types of diversity (alpha, beta, and gamma) have a scale, heterogeneity and homogeneity can be interchangeable with diversity that is measured spatially.

\section{Heterogeneity is affected by multiple factors}

Identification of heterogeneity will vary with decisions about study design and the response variable of interest, such as the scale of comparison (i.e., spatial sampling units) and hierarchical levels of organization (e.g., individuals, populations). For example, historically in North America, frequent surface fires were heterogeneous in space but produced low tree species diversity and a simple overstory canopy structure in oak- or pine-dominated ecosystems (Hanberry et al., 2014). However, even though tree species' richness and evenness were low, there was a wide range of tree densities from savannas to closed woodlands across the landscape, and richness of herbaceous plants and animals associated with light increased. Similarly, but without disturbance, old growth eastern forests of sugar maple-American beech contained low tree species diversity, but contained a high diversity of unique species dependent on complex tree structure. These forest ecosystems were both heterogeneous and homogenous. 
In addition, the type, severity, and extent of a disturbance process may reverse identification of heterogeneity or homogeneity. For example, Kolasa and Rollo (1991) pointed out that initially, disturbances produce heterogeneity in the landscape but as the extent of disturbances increases beyond $50 \%$ of the area, the matrix may be of a new type and become increasingly homogenous. Abiotic factors and biotic organisms will also influence disturbances and may increase or decrease measurements of heterogeneity.

\section{Heterogeneity as a value-laden term}

Heterogeneity is considered a desirable attribute, but heterogeneity depends on what is measured. A landscape composed of only pines or oaks may not be heterogeneous in measurements of tree richness in space, but still is valuable. Unexpected implications (when heterogeneity is considered desirable) of the term heterogeneity (e.g., Geri et al., 2010) can occur when heterogeneity due to multiple land uses may indicate the detrimental condition of fragmentation rather than a range of vegetation stages and types beneficial to wildlife and plants. Heterogeneity can be undesirable when representative of invasive species or intensive land use (e.g., diversity may not be greater in a heterogeneous landscape that is predominantly agricultural or urban and heterogeneity may increase due to invasive species). Thus, heterogeneity does not necessarily connote a positive situation and likewise, homogeneity does not imply a negative situation.

\section{Conclusions}

Generalizations about heterogeneity can be misleading and meaningless without context (Wiens, 2000). Ambiguity can be resolved by coordination in the concept and application of the terms heterogeneity and homogeneity. To be classified as heterogeneous, the variable should be responsive to primary sources of variation, such as environmental gradients. At the very least, researchers who use the term heterogeneity should define all aspects of heterogeneity to prevent ambiguous terminology from creating confusion. What are the units of comparison? Which abiotic gradients, biotic organisms, biotic level of organization (e.g., genetic, assemblage), processes, structure, or landscape metrics vary among units? Are the response variables (heterogeniables?) a secondary response to primary sources of variation? In some cases, use of the term variation rather than heterogeneity may be clearer and less complicated by value judgments (i.e., the notion that increased heterogeneity is better).

Edited by: J. M. Halley

Reviewed by: two anonymous referees

\section{References}

Fahrig, L., Baudry, J., Brotons, L., Burel, F. G., Crist, T. O., Fuller, R. J., Sirami, C., Siriwardena, G. M., and Martin, J. L.: Functional landscape heterogeneity and animal biodiversity in agricultural landscapes, Ecol. Lett., 14, 101-112, 2011.

Geri, F., Amici, V., and Rocchini, D.: Human activity impact on the heterogeneity of a Mediterranean landscape, Appl. Geogr., 30, 370-379, 2010.

Hall, L. S., Krausman, P. R., and Morrison, M. L.: The habitat concept and a plea for standard terminology, Wildlife Soc. B., 25, 173-182, 1997.

Hanberry, B. B., Jones-Farrand, D. T., and Kabrick, J. M.: Historical open forest ecosystems in the Missouri Ozarks: Reconstruction and restoration targets, Ecol. Restor., 32, 407-416, 2014.

Hutchings, M. J., John, E. A., and Stewart, A. J. A.: The ecological consequences of environmental heterogeneity, Blackwell, Malden, MA, USA, 452 pp., 2000.

Kolasa, J. and Pickett, S. T. A.: Ecological heterogeneity, Springer, New York, 332 pp., 1991.

Kolasa, J. and Rollo, C. D.: The heterogeneity of heterogeneity, in: Ecological Heterogeneity, edited by: Kolasa, J. and Pickett, S. T. A., Springer, New York, USA, 1-23, 1991.

Lausch, A., Pause, M., Doktor, D., Preidl, S., and Schulz, K.: Monitoring and assessing of landscape heterogeneity at different scales, Environ. Monit. Assess., 185, 9419-9434, 2013.

Li, H. and Reynolds, J. F.: On definition and quantification of heterogeneity, Oikos, 73, 280-284, 1995.

Lindenmayer, D. B. and Fischer, J.: Tackling the habitat fragmentation panchreston, TREE, 22, 127-132, 2007.

Madin, J. S., Bowers, S., Schildhauer, M. P., and Jones, M. B.: Advancing ecological research with ontologies, Trends. Ecol. Evol., 23, 159-168, 2008.

Malanson, G. P. and Cramer, B. E.: Landscape heterogeneity, connectivity, and critical landscapes for conservation, Divers. Distrib., 5, 27-39, 1999.

Marsh, M. K., Hutchings, M. R., McLeod, S. R., and White, P. C. L.: Spatial and temporal heterogeneities in the contact behaviour of rabbits, Behav. Ecol. Sociobiol., 65, 183-195, 2011.

McInerny, G. J. and Etienne, R. S.: Stitch the niche - a practical philosophy and visual schematic for the niche concept, J. Biogeogr., 39, 2103-2111, 2012.

McIntosh, R. P.: Concept and terminology of homogeneity and heterogeneity, in: Ecological Heterogeneity, edited by: Kolasa, J. and Pickett, S. T. A., Springer, New York, 24-46, 1991.

Olden, J. D. and Rooney, T. P.: On defining and quantifying biotic homogenization, Global Ecol. Biogeogr., 15, 113-120, 2006.

Pickett, S. T. A., Cadenasso, M. L., and Jones, C. G.: Ecological heterogeneity: an ontogeny of concepts and applications, in: The ecological consequences of environmental heterogeneity, edited by: Hutchings, M. J., John, E. A., and Stewart, A. J. A., Blackwell, Malden, MA, USA, 33-52, 2000.

Rees, M., Mangel, M., Turnbull, L., Sheppard, A., and Briese, D.: Ecological heterogeneity: an ontogeny of concepts and applications, in: The ecological consequences of environmental heterogeneity, edited by: Hutchings, M. J., John, E. A., and Stewart, A. J. A., Blackwell, Malden, MA, 237-266, 2000. 
Rosenberg, D. B. and Freedman, S. M.: Temporal heterogeneity and ecological community structure, Int. J. Environ. Stud., 46, 97102, 1994.

Stein, A., Gerstner, K., and Kreft, H.: Environmental heterogeneity as a universal driver of species richness across taxa, biomes and spatial scales, Ecol. Lett., 17, 866-880, 2014.

Tsunoda, T., Kachi, N., and Suzuki, J.-I.: Availability and temporal heterogeneity of water supply affect the vertical distribution and mortality of a belowground herbivore and consequently plant growth, PLoS ONE, 9, e100437, doi:10.1371/journal.pone.0100437, 2014.
Weins, J. A.: Ecological heterogeneity: an ontogeny of concepts and applications, in: The ecological consequences of environmental heterogeneity, edited by: Hutchings, M. J., John, E. A., and Stewart, A. J. A., Blackwell, Malden, MA, USA, 9-32, 2000. 